\title{
Cintilação em Ondas Milimétricas na Faixa de Absorção do Oxigênio -Funções Densidade Espectral Temporal
}

\author{
Luiz A. R. da Silva Mello e Carlos Gustavo S. Migliora
}

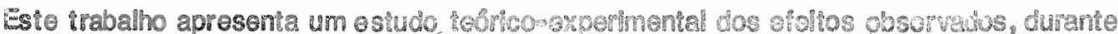

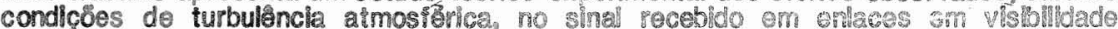
oporando em freqüenclas sujolias à absorço por gases na fakxa de ondas mithemericas. E desorvolvido um modelo teórico que pormhte caracterizar \& chnthilagåo em termos

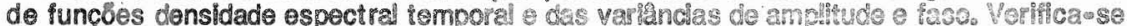

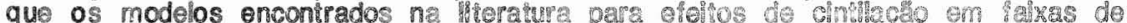
froquêencia om que o mecanismo de absorcaro pode ser desprezado constutuomose num caso particular da prescnte toorlat.

Os resultados teóficos sto comparados com medidas de flutuacôes de amplinde - fase diferenclal realizadas em dois enlaces experimentals oporando nas trocusholas

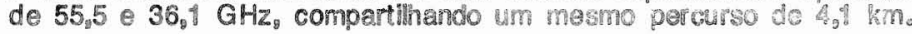

\section{In:rodução}

O sinal recebido num enlace eletronagnótico on visiblidade é composto por um campo módio ou coerente a uma componente flutuante, ou campo incoerente. O campo coerente depende das caracteristicas médias do melo de propagação onquanto que o campo incoerente estå associado às variacöes aleatorias espaço-temporais dos parknotios do maio (turbulência). A intensidade das fluruaçöes, comumente donorihadas chilaçōes, da amphitude $\theta$ da fase do sinal recobido, dopsndom da Pragẹêneia de propagaçäo, da distância entre o transmissor a o recsptor a das condiçỏes da troposiera. Na faixa de microondas o efeito de cinhlacho pode, on geral, ser desprezado, mas representa una séria limitaçäo ao projeto de enlaces rádio em visibilidade oporando om frequiências ósicas.

Na faixa de ondas milimétricas, em condịōes de furbulência atmos?érica, a cintilação observada em ligacóes em visibilidade $\delta$ suficientemente untensa para merecer um estudo detalhado, näo só para a avaliação de sous efeltos no desempenho de sistemas, como tambúm por representar ume ferramenta importante em técnicas de sensopiamento remoto[i]- [5].

Os autores sז̊ Professores do Contro de Estudos em Talocomunicaçes da PUChalo. 22453. Aio de Janeiro, Ris. 
A turbulência atmosférica é caracterizada pela formação e movimentação de massas de ar sob diferentes condiçōes de temperatura e pressão e com diferentes velocidades de deslocamento, provocadas pelo aquecimento não uniforme da troposfera, pela ação do sol e do solo, e pela ação do vento. Numa atmosfera turbulenta o índice de refração comporta-se como um campo aleatório variante no tempo.

Os fundamentos da teoria de propagação em meíos aleatórios foram estabelecidos por Chernov[6]e Tatarski[7] [8]. Estes resultados ioram posteriormente refinados por Ishimaru [9] - [11], que estendeu os tratamentos anteriores, limitados ao caso da propagaçáo de ondas planas, para incluir os casos de ondas esféricas e feixes colimados, sonvergentes e divergentes. Todos əstes trabalhos desprezavam o efeito da absorção por gases, considerando o índice de refração da atmosfera como um campo aleatório real.

A influência da absorção foi introduzida inicialmente por Izyumov [12], que resolveu a equação de onda para um índice de refração complexo. Gurvich [13]obteve soluções assintóticas para as densidades espectrais temporais das flutuações de amplitude e de iase de uma onda monocromática, problema também tratado por Armand et alli [14], Ott[15],e Ott e Thompson[16].Estes últimos obtiveram ainda soluções numéricas para as integrais que representam estas funções. Mais recentemente, um tratamento teórico bastante consistenìe, porém limitado à determinação das funções correlação espacial das flutuações de amplitude e de fase, foi apresentado por Manning et alli [17].

$\mathrm{Na}$ área experimental, Janes et alli [18] realizaram medidas da densidade espectral de amplitude e rase em 9,6 e $34,5 \mathrm{GHz}$. Trabalho semelhante foi desenvolvido por Ho et alli [19] em 36 e $110 \mathrm{GHz}$, para as ?lutuações de amplitude. Em freqüências sujeitas à absorção por gases, os principais resultados experimentais são devidos a. Armand et alli [20], que realizaram medidas na faixa de $60 \mathrm{GHz}$ e a Jayasuryia et alli [21] e Medeiros Filho et alli[22], na faixa de $55 \mathrm{GHz}$.

Neste trabalho, a formulação de Ishimaru[10]é estendida de modo a incluir os efeitos da absorção por gases atmosféricos, com a suposição de que o índice de refração da troposfera é um campo aleatório complexo variante no tempo. A partir da solução da equação de onda pelo método de Rytov, a utilizando iepresentações espectrais para as flutuações das partes real e imaginária do índice de refração, são obtidas as funções correlação cruzada em espaço e treqüência da log-amplitude e fase da onda propagante, num desenvolvimento semelhante ao apresentado no trabalho de Manning et alli [17].Através da aplicação da hipótese do congelamento de Taylor [10], estas funções são convertidas do domínio espacial para o temporal. A tansformada de Fourier, em relação à variável tempo, das funções correlação cruzada em freqüência e tempo resultantes fornece funções densidade coespectral 
temporal das flutuações de amplitude e de fase. As soluções analíticas obtidas são gerais e reproduzem resultados particulares obtidos por integração numérica[16] bem como a solução de Ishimaru[10] para o caso sem perdas quando se faz a parte imaginária do indice de reiração igual a zero. Além disso, as aproximações assintóticas destas soluções coincidem com os resultados encontrados na literatura [13]-[16].

Os resultados téricos obtidos são ainda comparados com dados, obtidos om medidas de amplitude e fase diferencial realizadas em dois enlaces experimentais, compartilhando um mesmo percurso, operando nas freqüências de $55,5 \mathrm{GHz}$, na banda de absorção do oxigênio, e $36,1 \mathrm{GHz}$, uma janela de absorção do espectro. Para permitir a comparação entre resultados teóricos e experimentais foram realizadas, paralelamente às medidas de amplitude e lase do sinal recebido em cada enlace, medidas de parâmetros meteorológicos: temperaturas de bulbo seco e bulbu úmido, pressão barométrica, velocidade e direção do vento.

\section{Caracterização da Atmosiera}

Em problemas de propagação eletromagnética na faixa de microondas a atmosfera é usualmente inodelada por um índice de refração real que varia com a altura mas é constante na direção horizontal no plano do enlace. Em faixas de ondas milimétricas sujeitas à absorção por gases, os efeitos observados no sinal recebido podem ser explicados modelando-se o índice de refração da troposfera na forma

$n(r, t, f)=1+n_{1}(r, f, t)+i\left[m_{0}(r, f, t)+m_{1}(r, f, t)\right]$

onde $r$ é o vetor posição $e$

$\left.m_{0}, i r, f, t\right)=\langle\operatorname{lm}[n(r, f, t)]\rangle$

com Im<•>denotando a parte imaginária da quantidade entre colchetes e $<\cdot>$ denotando a média espacial no volume considerado. Os termos $n_{1} \in m_{1}$ representam as flutuações das partes real e imaginária do índice de refração em condições de turbulência atmosférica.

O termo mo está associado à atenuação provocada pela absorção por gases. A Fig. 1 mostra curvas da atenuação específica devida ao oxigênio e ao vapor d'água de uma onda plana em propagação numa atmosfera homogênea em condições normais de temperatura e pressão. As faixas de frequêencias em que a atenuação específica totai é muito reduzida são denominadas janelas atmosféricas, e a parte imaginária do índice de refração pode ấ 


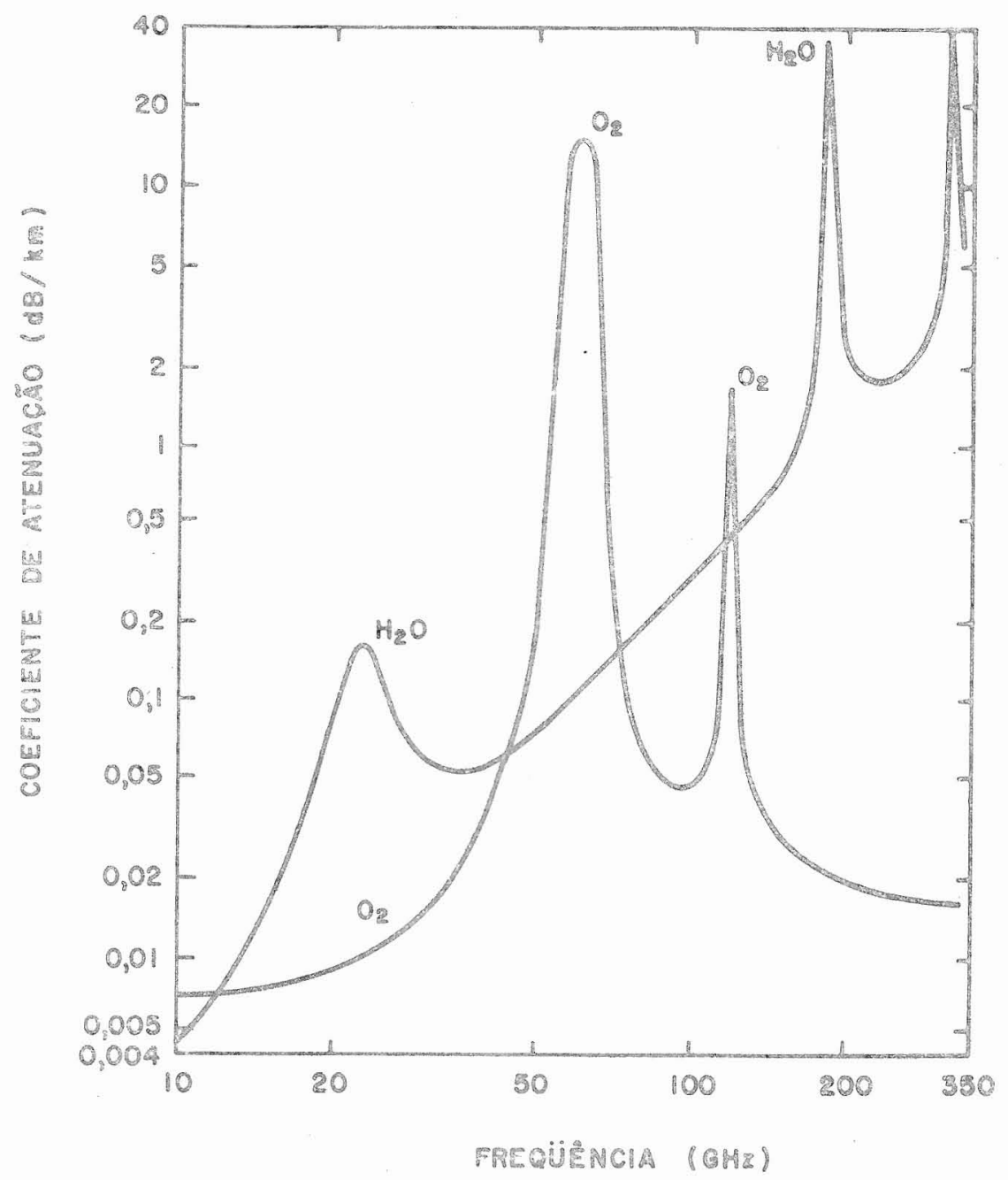

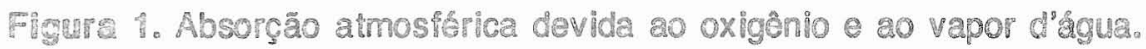

Ser desprozada $\left(m_{0} \cong 0, m_{1} \cong 0\right.$. Nas demais regibes do espectro o comportamento de mo é, em geral, caracterizado por modelos emplricos. Neste trabalho, para freqüências em torno do pico de absorģão pelo oxigênio, on $60 \mathrm{GHz}$, será empregado o modelo de Ott a Thompson [21], segundo o qual

$$
m_{0}(f, t)=\frac{20,4624 \times 10^{-6}}{15,3664+(60-f)^{2}}\left[\frac{P_{d}}{P_{0}}\right]^{x(f)}\left[\frac{T_{0}}{T}\right]^{y(f)}
$$


onde $\uparrow$ é a frequência em $\mathrm{GHz}, \mathrm{P}_{d}$ a pressão média do ar seco em mbar, $T$ a temperatura média em Kelvin $(K), P_{0}=1013,25$ mbar e $T_{0}=300 K$. Os expoentes $x(f)$ e $y(f)$ são parâmetros cujos valores foram determinados ampiricamente por Ott a Thompson[23] para algumas \&reqüências.

No desenvolvimento a ser apresentado na Seçäo 3 seräo utilizadas representaçöes espectrais bidimensionais [12] para os termos de flutuação n1 e m1. Admitindo-se que o indice de rêrraçăo da atmosfera é um campo aleatório homogêneo e isotrópico, estas representaçôes têm a forma

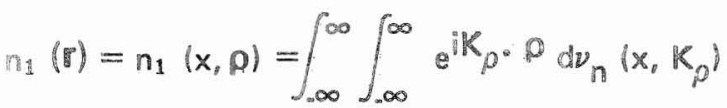

$m_{1}(\mathbb{r})=m_{1}(x, \rho)=\int_{-\infty}^{\infty} \int_{-\infty}^{\infty} e^{i K_{\rho} \cdot \rho} d y_{m}\left(x, K_{\rho}\right)$

onde $K_{\rho}=K_{y} \hat{y}+K_{z} \tilde{z}, \rho=y \hat{y}+z \underline{z}$ e $y=x \hat{x}+\rho$, com $\hat{x}, \hat{y}$ e z denotando os vetores unitários nas direçóes $x, y, z \in K_{y}$ e $K_{z}$ denotando as Irequências espaciais de flutuação nas direções y $e z$. A dependência de $n_{1}$ e $m_{1}$ com a frequêencia e 0 lempo fol, por simplicidade, omitida em (4) $\theta(5)$.

As funçōes correlação das amplitudes espectrais aleatórias $d u_{n}$ e $d v_{m}$ atendem a relações da forma

$\left.<d v_{j}\left(x^{2}, K_{\rho_{1}}\right) d \nu_{j}\left(x^{\prime \prime}, K_{\rho_{2}}\right)>=F_{i j}|| x^{0}-x^{i n} \mid, K_{\rho_{1}}\right) \delta\left(K_{\rho_{1}}-K_{\rho_{2}}\right)$

$d K_{\rho_{1}} d K_{\rho_{2}}$

para $i=n, m$ e $j=n, m$. As funçöes $F_{i j}$ são denominadas densidades espectrais bidimensionais e relacionam-se com as densidades espectrais tridimensionais $\Phi_{\text {Ij }}(K)$ através da relação

$\Phi_{i j}(K)=\frac{1}{2 \pi} \int_{-\infty}^{\infty} \exp \left(i K_{x} x\right) F_{i j}\left(|x|, K_{\rho}\right) d x$

para $x=x^{\prime}-x^{\prime \prime}, K=K_{x} \tilde{x}+K_{p}, K=|K|, i=n$, $m$ e $j=n, m$, com $K_{x}$ denotando a Preqüência espacial de flutuação na direção $x$. É comumente aceiro que, an condições de turbulência atmosiérica, as flutuações das partes real e maginária do indice de refraçäo podem ser caracterizadas pelo espectro de Kolmogorov [24], dado por

$\Phi_{n n}(K)=\Phi_{n}(K)=C_{n}^{2} K^{-11 / 3}$ 


$$
\begin{aligned}
& \Phi_{m m}(K)=\Phi_{m}(K)=C_{m}^{2} K^{-11 / 3} \\
& \Phi_{m n}(K)=\Phi_{n m}(K)=C_{n m} K^{-11 / 3}
\end{aligned}
$$

para $2 \pi / L_{0}<K<2 \pi / l_{0}$, onde $L_{0}$ e $\ell_{0}$ são, respectivamente, os tamanhos de escala externo e interno da turbulência. Esta faixa de valores de $\mathrm{K}$ é denominada sub-faixa inercial.

As constantes de estrutura das partes real $\left(C^{2} n\right)$ e imaginária $\left(C^{2} m\right)$ do indice de refração e a constante de estrutura cruzada $\left(\mathrm{C}_{n m}\right)$ dependem do comportamento estatístico da pressão, temperatura e pressão de vapor d'água na atmosfera. Seguindo o modelo de Hill et alli[25],e utilizndo dados de medidas, Medeiros Filhos[26] obteve as seguintes relações semi-empíricas entre as constantes de estrutura

$$
\begin{aligned}
& \frac{\mathrm{C}_{\mathrm{m}}^{2}}{\mathrm{C}_{\mathrm{n}}^{2}}=\nu^{2}\left[\frac{1+0,4 \sigma_{\mathrm{eT}} / \sigma_{\mathrm{T}}^{2}+0,04 \sigma_{\mathrm{e}}^{2} / \sigma_{\mathrm{T}}^{2}}{1-7,0 \sigma_{\mathrm{eT}} / \sigma_{\mathrm{T}}^{2}+12,25 \sigma_{\mathrm{e}}^{2} / \sigma_{\mathrm{T}}^{2}}\right] \\
& \frac{\mathrm{C}_{\mathrm{nm}}}{\mathrm{C}_{\mathrm{n}}^{2}}=\nu\left[\frac{1-3,3 \sigma_{\mathrm{eT}} / \sigma_{\mathrm{T}}^{2}-0,7 \sigma_{\mathrm{e}}^{2} / \sigma_{\mathrm{T}}^{2}}{1-7,0 \sigma_{\mathrm{eT}} \bar{T}_{\mathrm{T}}^{12}+12,25 \sigma_{\mathrm{e}}^{2} / \sigma_{\mathrm{T}}^{2}}\right] \\
& \nu=1,93 \times 10^{4} \mathrm{~m}_{\mathrm{o}}\left[\frac{\langle\mathrm{T}\rangle}{\langle\mathrm{P}\rangle+9620\langle\mathrm{e}\rangle / \overline{\mathrm{T}}\rangle}\right]
\end{aligned}
$$

onde $\sigma_{\mathrm{e}}^{2}, \sigma_{T}^{2},<\mathrm{e}>\mathrm{e}<T>$ são respectivamente as variâncias e médias da pressão de vapor d'água e da temperatura absoluta, $\sigma_{\text {eT }}$ a covariância entre estas duas variáveis, $\mathrm{e}<\mathrm{P}>0$ valor médio da pressão na atmosfera.

\section{Funções Correlação Cruzada das Log-Flutuações de Amplitude e das Flutuações de Fase}

Numa atmosfera turbulenta a constante dielétrica é um campo aleatório variante no tempo $\varepsilon_{r}(r, t)$. Expressando o campo elétrico associado a uma onda em propagação neste meio na forma

$E(\mathbf{r}, t)=E^{\prime}(\mathbf{r}, t) \exp (-i 2 \pi f t)$ 
onde $f$ é a freqüência de propagação e a dependência espaço-temporal de $E^{\prime}(r, t)$ está associada ao comportamento aleatório do meio, a equação de onda se escreve na forma

$\nabla^{2} E^{\prime}+k^{2} \epsilon_{r} E^{\prime}=\frac{1}{c^{2}} \frac{\partial^{2}}{\partial t^{2}}\left(\epsilon_{r} E^{\prime}\right)-2 i \frac{k}{c} \frac{\partial}{\partial t}\left(\epsilon_{r} E^{\prime}\right)-\nabla\left[E^{\prime} . \nabla\left(l_{n} \epsilon_{r}\right)\right]$

onde $c$ é a velocidade de propagação da onda e $k=2 \pi f / c$. Os termos envolvendo derivadas em relação ao tèmpo de $\varepsilon_{\mathrm{r}}$ E podem ser desprezados na equação acima desde que se tenha

$\epsilon_{\mathrm{r}} \gg \frac{1}{(2 \pi f)^{2}} \frac{\partial^{2} \epsilon_{\mathrm{r}}}{\partial \mathrm{t}^{2}}-\mathrm{i} \frac{\partial \epsilon_{\mathrm{r}}}{\partial \mathrm{t}}$

Isto equivale a considerar que a taxa de variạção temporal da estrutura da troposfera é muito pequena em relação à freqüência de oscilação da onda, o que efetivamente ocorre para a propagação na faixa de ondas milimétricas. Além disto, pode ser demonstrado [17] que o último termo da equação, denominado termo de despolarização, pode ser desprezado quando o comprimento de onda é muito menor que o tamanho de escala interno da turbulência $2_{0}$ que, para a atmosfera, é da ordem de $1 \mathrm{~cm}$. Assim, para freqüências na faixa de ondas milimétricas ou superiores, a equação de onda pode ser aproximada por

$\nabla^{2} E^{\prime}(\mathbf{r})+k^{2} n^{2}(\mathbf{r}) E^{\prime}(\mathbf{r})=0$

onde $n(r)=\sqrt{\varepsilon_{r}(\mathbf{r})}$ é dado por (1).

Considerando a transmissão de uma onda plana linearmente polarizada, a equação escalar de onda correspondente pode ser resolvida pelo método de Rytov, expressando o fasor campo elétrico na forma

$E^{\prime}(r)=\exp \left[\psi_{0}+\psi_{f}\right]$

onde $\exp \left(\psi_{0}\right)$ representa o campo na ausência de turbulência e $\exp \left(\psi_{f}\right)$ representa um termo aleatório associado às flutuações do índice de refração. A primeira aproximação de $\psi_{f}$, denotada $\psi_{1}$, obtida pelo método das perturbaçổes suaves é da forma [17]

$\psi_{1}^{\prime}=\chi+i S_{1}$

com 


$$
\begin{aligned}
& (k, x, \rho)=\int_{0}^{x} d x^{\prime} \int_{-\infty}^{\infty} \int_{-\infty}^{\infty}\left[H_{\Omega}\left(x^{2}, k_{,} K_{p}, \rho\right) d y_{n}\left(x^{\prime}, K_{p}\right)-\right. \\
& \left.H_{2}\left(x^{0}, k, K_{\rho}, \rho\right) d v_{m}\left(x^{0}, K_{\rho}\right)\right] \\
& \left.i_{1} \mid k, x, p\right)=\int_{0}^{x} d x^{\prime} \int_{-\infty}^{\infty} \int_{-\infty}^{\infty}\left[H_{1}\left(x^{0}, k, K_{\rho}, p\right) d \nu_{m}\left(x^{\prime}, K_{\rho}\right)+\right. \\
& \left.H_{2}\left(x^{\prime}, k_{,} k_{\rho}, \rho\right) d v_{n}\left(x^{\prime}, k_{0}\right)\right]
\end{aligned}
$$

nde

$H_{1}\left(x^{\prime}, k_{,} K_{\rho}, \rho\right)=e^{i K_{p} \cdot \rho \operatorname{Re}\left[H\left(x^{\prime}, k, K_{\rho}\right)\right]}$

$I_{2}\left(x^{3} K_{,} K_{\rho}, \rho\right)=e^{i K_{\rho} \cdot P} \operatorname{lm}\left[H\left(x^{0}, K_{,} K_{\rho}\right)\right]$

$f\left(x^{\prime}, k_{s} k_{p}\right)=i k \exp \left[\frac{-i k_{p}^{2}\left(x-x^{\prime}\right)}{2 k\left(1+i m_{0}\right)}\right]$

Inde foram empregadas representaçōes nas lormas apresentadas em (4) - (5) para as tlutuações das partes real e imaginária do indice de refração.

A funçăo $\chi$ é denominada log-flutuação de amplitude do sinal e é igual zo logaritmo da razão entre as amplitudes do campo em condições de turbuência atmosférica e numa atmosfera homogênea. Analogamente, $S_{1}$ é a diferença entre as lases dos campos nestas duas situaçöes.

As funções correlação cruzada em espaço e frequência de $\bar{\chi}$ e $S_{1}$ são defenidas por

$B_{\chi}\left(k_{1}, k_{2}, p_{1}, r_{2}\right)=\left\langle\chi\left(k_{1}, x_{1}, p_{1}\right) \chi\left(k_{2}, x_{2}, p_{2}\right)>\right.$

$B_{S}\left(k_{1}, k_{2}, \mathbb{P}_{1}, \mathbb{P}_{2}\right)=\left\langle S_{1}\left(k_{1}, x_{1}, p_{1}\right) S_{1}\left(k_{2}, x_{2}, p_{2}\right)\right\rangle$

De (20) até (26), empregando (6) e (7) e a transformação de variáveis $\eta=\left(x_{1}+x_{2}\right) / 2$ a $\xi=x_{1}-x_{2}$ com as aproximaçóes discutidas em [12]. thega-se a

$B_{S}\left(k_{1}, k_{2}, \rho_{1}, \rho_{2}\right)=2 \pi^{2} \int_{0}^{\infty} d_{0}^{\infty} \int_{0}^{\infty} J_{0}\left(k_{0}\right) H\left(\eta, k_{1}, K\right) H\left(\eta_{1}, k_{2}, K\right) \cdot$ 


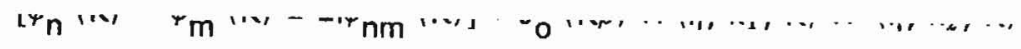

$$
\begin{aligned}
& \cdot\left[\phi_{n}(K)+\phi_{m}(K)\right] K d K \\
& \text { onde } \rho=\left|\rho_{1}-\rho_{2}\right| \text { e } J_{0} \text { é a função de Bessel de ordem zero. }
\end{aligned}
$$

No desenvolvimento até aqui apresentado, a dependência temporal do índice de refração foi desprezada. Entretanto, na maioria dos estudos experimentais, são realizadas medidas da variação temporal do sinal recebido, por sua relativa simplicidade em comparação com a medida de características espaciais. Assim, é necessário realizar uma conversão de espaço para tempo. Isto pode ser feito através da hipótese do congelamento de Taylor, segundo a qual as variações do meio durante o intervalo de observação são devidas unicamente a um deslocamento da estrutura turbulenta em relação à linha de visada do enlance, pela ação do vento. A utilização desta hipótese baseiase no fato de que as variaçōes de velocidade do vento são pequenas em relação à sua velocidade média [9] e corresponde a assumir para o índice de refração um comportamento da forma

$n\left(r, t=t_{1}\right)=n\left(r-v t_{1}, t=0\right)$

onde $\mathbf{v}=\mathbf{v}_{\mathbf{y}} \hat{\mathbf{y}}+\mathbf{v}_{\mathbf{z}} \mathbf{z} e ́$ a velocidade do vento na direção transversal à linha de visada do enlace. Esta suposição não invalida o desenvolvimento até aqui apresentado, desde que a relação dada por (16) seja atendida. Neste caso, as representações dadas por (27) podem ser empregadas para fornecer as correlaçōes cruzadas de log-amplitude e fase no mesmo ponto do espaço $\left(\eta, \rho_{\mathrm{o}}\right)$ mas em diferentes instantes de tempo $\tau_{1}$ e $\tau_{2}$, fazendo-se $\rho_{1}=\rho_{\mathrm{o}}$ $+v \tau_{1}$ e $\rho_{2}=\rho_{0}+v \tau_{2}$. Escolhendo, por conveniência, $\rho_{0}=0$ e realizando a integração em $\eta$ nas equações (27) tem-se, para $\tau=\tau_{1}-\tau_{2}$

$\mathrm{B}_{\mathrm{S}}^{\mathrm{X}}\left(\mathrm{k}_{1}, \mathrm{k}_{2}, \tau\right)=2 \pi^{2} \mathrm{k}_{1} \mathrm{k}_{2} L \int_{0}^{\infty}\left[f_{1}\left(k_{1}, k_{2}, K\right) \phi_{\mathrm{n}}(K)+\underset{2}{f_{2}}\left(k_{1}, k_{2}, K\right) \phi_{m}(K) \mp\right.$

$\left.\mp f_{3}\left(k_{1}, k_{2}, K\right) \phi_{n m}(K)\right] J_{0}(K v \tau) K d K$

onde $L$ é o comprimento do enlace, $v=|\mathbf{V}|$ e as funções filtro $f$ são dadas por

$f_{1}\left(k_{1}, k_{2}, K\right)=\frac{1}{L K^{2}} \cdot R e\left\{\frac{1}{a}\left[e^{a L K^{2}}-1\right] \mp \frac{1}{b}\left[e^{b L K^{2}}-1\right]\right\}$

Revista da Sociedade Brasileira de Telecomunicações

Volume 2, N. 1, dezembro 1987. 
$f_{3}\left(k_{1}, k_{2}, K\right)=\frac{2}{L K^{2}} \operatorname{lm}\left\{\frac{1}{b}\left[e^{b L K^{2}}-1\right]\right\}$

com

$a=-\left[\frac{i}{k_{q}}+\frac{m_{a}}{k_{p}}\right]$

$b=\frac{-1}{k_{p}}\left(i+m_{o}\right)$

$m_{0}=\frac{m_{0}\left(k_{1}\right) k_{2}+m_{0}\left(k_{2}\right) k_{1}}{k_{1}+k_{2}}$

$k_{q}=\frac{2 k_{1} k_{2}}{k_{2}-k_{1}}$

$k_{p}=\frac{2 k_{1} k_{2}}{k_{2}+k_{1}}$

\section{Funções Densidade Coespectral de Potência}

As funções densidade coespectral de potência das log-flutuações de amplitude e das flutuações de fase do sinal recebido são as transformadas de Fourier das funções correlação cruzada definidas na seção anterior, sendo dadas por

${\underset{S}{X}}_{\chi}^{W_{1}}\left(k_{1}, k_{2}, \omega\right)=2 \int_{-\infty}^{\infty} \underbrace{B_{S}}_{S}\left(k_{1}, k_{2}, \tau\right) e^{i \omega \tau} d \tau$

Das expressões (29) a (36), empregando (8) a (10) e considerando a ordem de grandeza de mo, tem-se [27]

$$
\begin{aligned}
& \underset{S}{W_{\chi}}\left(k_{1}, k_{2}, \omega\right)=0,132 \pi^{5 / 2} k_{1} k_{2} L v^{5 / 3} \omega^{-14 / 3}\left\{\left(C_{n}^{2}+C_{m}^{2}\right) \cdot\right. \\
& {\left[\frac{A}{C^{2}} U_{1}\left(Z_{A}\right)+\frac{m_{0} B}{C^{2}}\left[\frac{\Gamma(7 / 3)}{\Gamma(17 / 6)}-U_{R}\left(Z_{A}\right)\right]\right] \mp}
\end{aligned}
$$


$\left.\mp\left(C_{n}^{2}-C_{m}^{2}\right) \frac{U_{1}\left(Z_{B}\right)}{B}, \pm \frac{C_{n m}}{B}\left[\frac{\Gamma(7 / 3)}{\Gamma(17 / 6)}-U_{R}\left(Z_{B}\right)\right]\right\}$

onde

$z_{A}=\left(m_{0} B-i A\right) \omega^{2}$

$Z_{B}=\left(m_{0} B-i B\right) \omega^{2}$

$U_{R}(Z)=\operatorname{Re}\left[e^{Z} U(1 / 2,-4 / 3, Z)\right]$

$U_{1}(Z)=\operatorname{Im}\left[e^{Z} U(1 / 2,-4 / 3, Z)\right]$

$A=L /\left(K_{q} v^{2}\right), B=L /\left(K p v^{2}\right)$ e $U$ é a função de Kummer cuja representação integral é dada por

$U(a, b, Z)=\frac{e^{Z}}{\Gamma(a)} \int_{1}^{\infty} e^{-Z t} t^{b-a-1}(t-1)^{a-1} d t$

A fim de visualizar o comportamento das soluções dadas por (37) é conveniente desenvolver aproximações para valores extremos de $\omega$. Duas regiões do espectro são de especial interesse e serão consideradas a seguir.

\section{a) Região de baixas freqüências}

$A \omega^{2}<B \omega^{2}<<1$

Aplicando-se expressões assintóticas da função de Kummer [28] em (37), tem-se [27] que, para $B \omega 2<<1, W \times\left(k_{1}, k_{2}, \omega\right)$ se escreve

$W_{\chi}^{O}\left(k_{1}, k_{2}, \omega\right)=k_{1} k_{2} L v^{5 / 3} \quad\left\{\left[1,69 C_{n}^{2}\left(B^{4 / 3} \operatorname{sen} \theta_{B}-\right.\right.\right.$

$\left.\left.-A C^{1 / 3} \operatorname{sen} \theta A^{\prime}\right)+2,19\left(2 C_{m}^{2}+m_{0} C_{n m}\right) \omega^{-8 / 3}\right\}$ 
enquanto $W_{S}\left(k_{1}, k_{2}, \omega\right)$ se escreve

$W_{S}^{0}\left(k_{1}, k_{2}, \omega\right)=2,19 k_{1} k_{2} L v^{5 / 3}\left(2 C_{n}^{2}-m_{0} C_{n m}\right) \omega^{-8 / 3}$

onde $\theta_{B}=7 / 3 \tan ^{-1}\left(-1 / m_{0}\right)$ e $\theta_{A}=7 / 3 \tan ^{-1}\left(-A / m_{0} B\right)$.

Para $k_{1}=k_{2}=k$ e $m_{0}=C^{2} m=C_{n m}=0$, (43) reduz-se ao resultado já obtido por Ishimaru [9] para o caso monocromático sem absorção.

\section{b) Região de altas freqüências}

$A \omega^{2}<<1, \ldots \quad B \omega^{2}>1$

A região de altas freqüências é definida desta forma, uma vez que, para bandas de freqüências de interesse prático, a região $A \omega^{2} \geqslant 1$ corresponde a freqüências de flutuação em que as densidades espectrais de potência são desprezíveis, confundindo-se com o nivel de ruído do equipamento receptor. Uma vez mais, empregando-se expressões assintóticas para a função de Kummer [28], obtém-se para $B \omega^{2}>>1$ e $A \omega^{2}<<1$,

$W_{\mathrm{S}}^{\infty}\left(k_{1}, k_{2}, \omega\right)=2,19 k_{1} k_{2} L v^{5 / 3}\left(C_{n}^{2}+C_{m}^{2}\right) \omega^{-8 / 3}$

Note-se que (44) coificicide com o resultado obtido por Ishimaru para o caso sem perdas, quando $\mathrm{C}_{\mathrm{m}}^{2}=0$.

O comportamento dos espectros de amplitude e fase, juntamente com surs aproximações assintóticas nas regiões de altas e baixas freqüências é mostrado nas figuras 2 até 4 . Em ambos os casos foram admitidos valores de $\mathrm{C}^{2} \mathrm{n}=1 \times 10^{-12}$, com $\mathrm{C}^{2} \mathrm{~m}=1 \times 10^{-12}$ e $\mathrm{C}_{\mathrm{nm}}=1 \times 10^{-15}$, comprimento de elance $L=10 \mathrm{~km}$ e velocidade transversal do vento $v=5 \mathrm{~m} / \mathrm{s}$. A Fig. 2 apresenta estas funções para $f_{1}=55 \mathrm{GHz}$ e $f_{2}=60 \mathrm{GHz}$. A Fig. 3 corresesponde a $f_{1}=f_{2}=60 \mathrm{GHz}$. Observa-se que, neste caso, as diferenças entre a solução exata e a aproximação dada pelas assíntotas é desprezível. A Fig. 4 mostra as mesmas funções para $f_{1}=f_{2}=36 \mathrm{GHz}$, freqüência em que a absorção por gases é desprezivel. A comparação entre os espectros das figuras 3 e 4 indica que o mecanismo de absorção não influencia o comportamento do espectro das flutuações de fase mas provoca um significativo aumento nas flutuações lentas de amplitude (região de baixas freqüências do espectro). Esta conclusão pode ser generalizada examinando-se as aproximações assintóticas do espectro dadas por (43) a (45). Uma vez que, na prática, $m 0 C_{n m}<<C^{2} m,<<C^{2} n$,quaisquer que sejam as características da turbulência, as assintotas de altas freqüências 


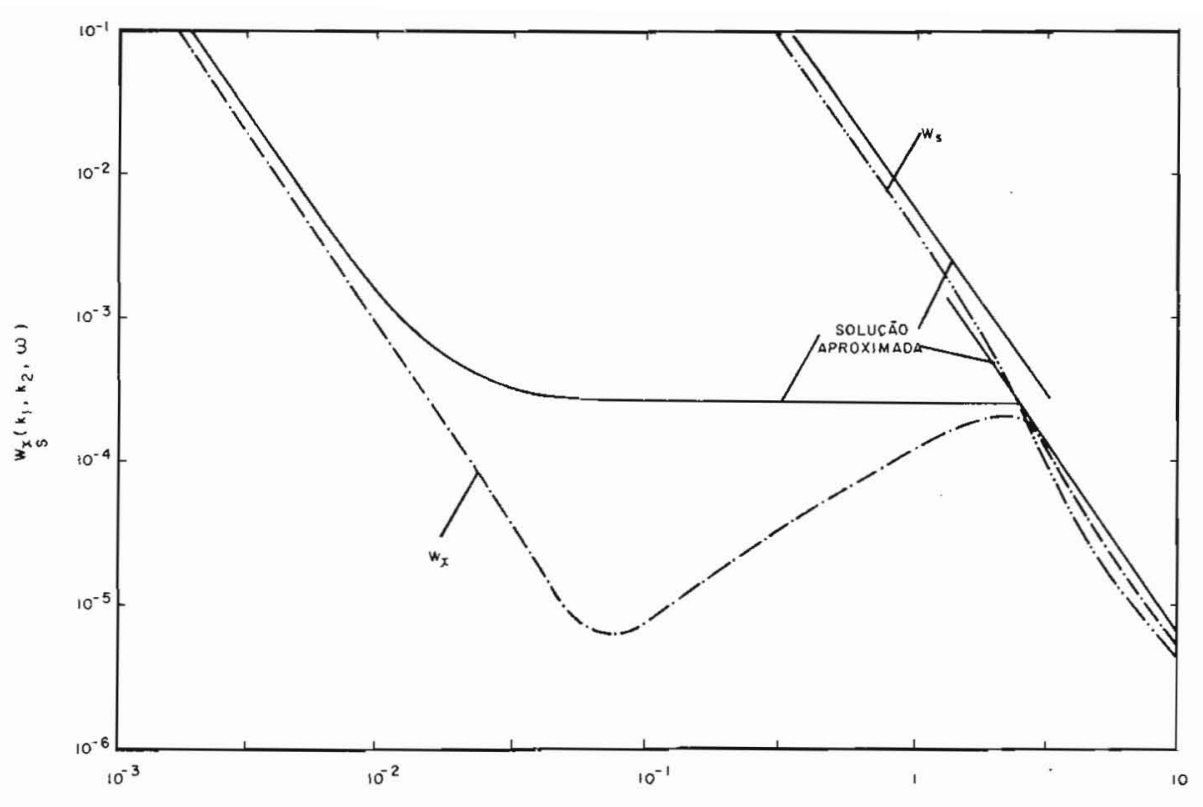

Flgura 2. Funções densidade espectral temporal para o caso com absorção $\left(f_{1}=55 \mathrm{GHz}, f_{2}=60 \mathrm{GHz}\right)$.

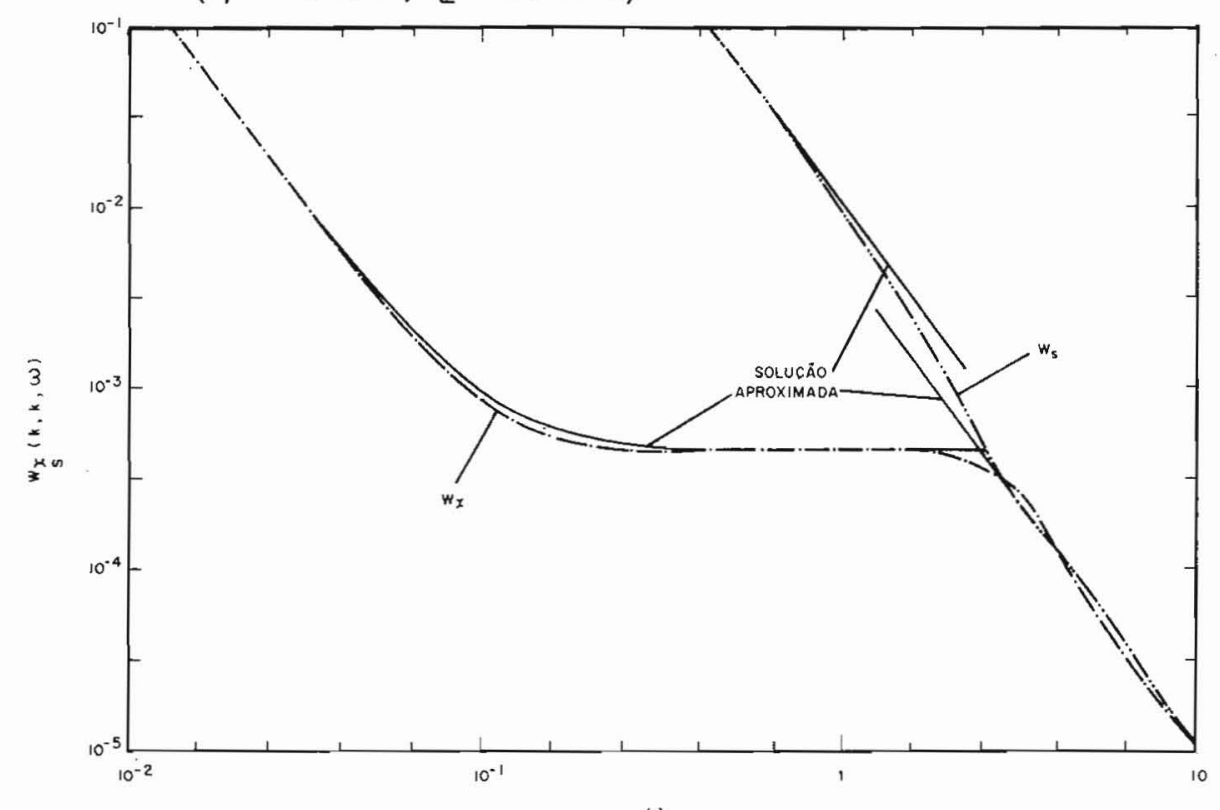

Figura 3. Funções densidade espectral temporal para o caso com absorção $\left(f_{1}=f_{2}=60 \mathrm{GHz}\right)$. 


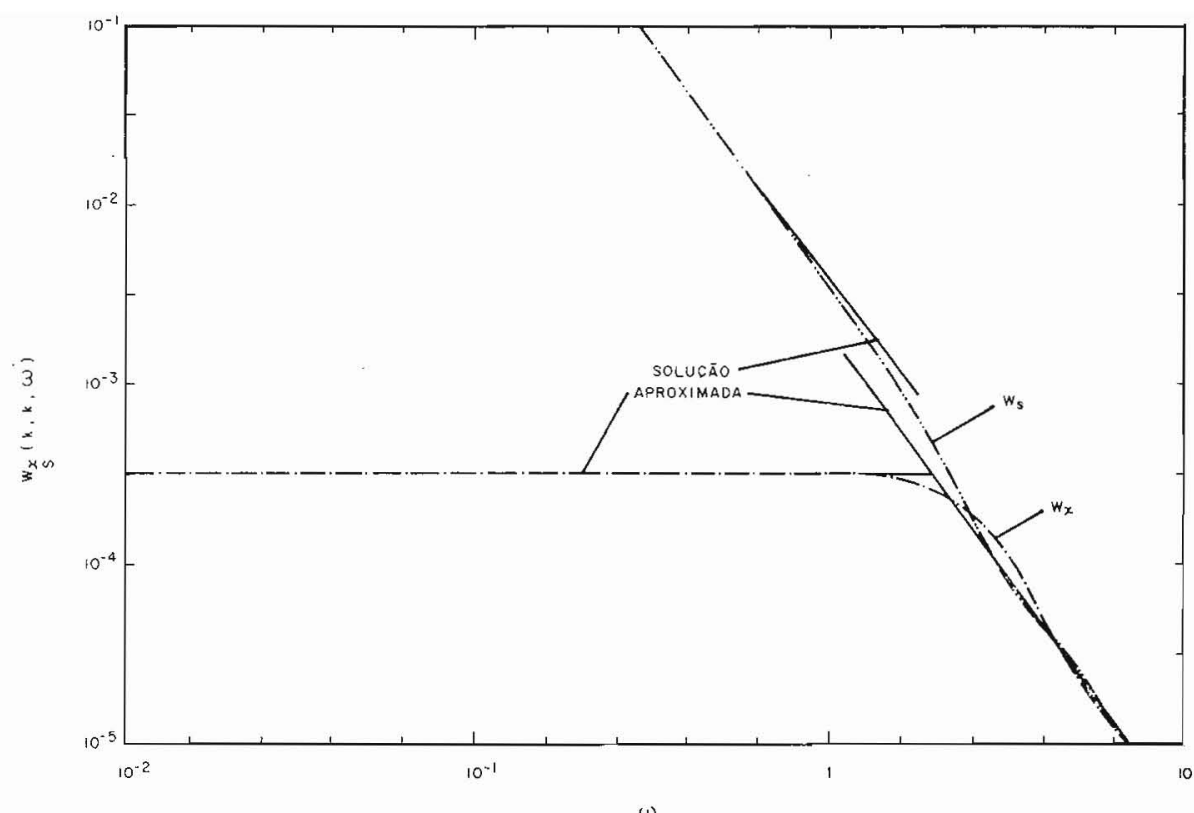

Figura 4. Funções densidade espectral temporal para o caso sem absorção $\left(f_{1}=f_{2}=36 \mathrm{GHz}\right)$.

dos espectros, dadas por (45), são essencialmente iguais para os casos com e sem absorção. $O$ mesmo é válido para as assíntotas de baixa freqüência dos espectros de fase. Para o espectro de log-amplitude, entretanto, a assíntota de baixa freqüencia, dada por $(43)$, é proporcional a $\omega^{-8 / 3}$ no caso com absorção, mas independe da freqüência de flutuaçăo para o caso sem absorção, em que $\mathrm{C}^{2} \mathrm{~m}=\mathrm{C}_{\mathrm{nm}}=\mathrm{mo}_{0}=0$.

\section{Resultados Experimentais}

As medidas cujos resultados são apresentados nesta seção foram realizadas em dois enlaces experimentais, operando na freqüência de $36,1 \mathrm{GHz}$ e na faixa de $55 \mathrm{GHz}$, compartilhando um mesmo percurso de $4,1 \mathrm{~km}$ entre 0 University College London e o Imperial College of Science and Technology. O terreno sob os enlaces é constituído parcialmente por uma área aberta, - Hyde Park, e parcialmente por áreas construídas. A altura das antenas é de cerca de $40 \mathrm{~m}$ em relação ao solo.

Estes resultados correspondem a très tipos de experimento:

i) medidas de amplitude: experimentos em que foram medidas as amplitudes 
dos sinais recebidos em $55,5 \mathrm{GHz}$ e $36,1 \mathrm{GHz}$, além dos parâmetros meteorológicos;

ii) medidas de fase diferencial: experimentos em que o sinal em $55 \mathrm{GHz}$ foi modulado em freqüência e medida a diferença de fase entre as bandas laterais do sinal resultante, a amplitude do sinal em $36,1 \mathrm{GHz}$ e os parâmetros meteorológicos;

iii) medidas de amplitude e fase diferencial: experimentos em que foram medidas as amplitudes das bandas laterais do sinal modulado em $55,5 \mathrm{GHz}$, sua diferença de fase e os parâmetros meteorológicos.

Cada experimento corresponde ao registro contínuo de dados, durante cerca de uma hora, em condiçōes de turbulência atmosférica. A Tabela 1 apresenta as condições de tempo, valores médios de parâmetros meteorológicos e as variâncias das diversas quantidades medidas para 12 exemplos extraídos do conjunto de experimentos realizados. A Tabela 1 apresenta ainda valores de $C \cdot \frac{2}{m} / C{ }_{n}^{2}$ e $C_{n m} / C_{n}^{2}$ estimados empregando-se as expressões em (3), (11), (12) e (13), e de $C{ }_{n}^{2}$. A constante de estrutura $C$ n poderia, em princípio, ser determinada a partir das medidas de parâmetros metecrológicos. Entretanto, considerando que as medidas eram do tipo pontual, referentes a um dos extremos do enlace, e certa limitação na precisão dos sensores utilizados, estimou-se $\mathrm{C}_{n}^{2}$ a partir da variância de log-amplitude $\left(\sigma_{\chi}^{2}\right)$ medida em 36 $\mathrm{GHz}$, pela relação [7], [12]:

$\sigma_{\chi}^{2}=0,307 C_{n}^{2} k^{7 / 6} L^{11 / 6}$

A Fig. 5 mostra as funções densidade espectrais das flutuações de log-amplitude das bandas laterais do sirial em $55 \mathrm{GHz}$, da diferença de fase entre estas bandas laterais e da log-amplitude em $36 \mathrm{GHz}$, relativas ao experimento 12, obtidas pela aplicação da transformada rápida de Fourier aos dados amostrados a cada 0,8 s. A Fig. 5 mostre ainda as assíntotas das diversas funçčes densidade espectral, obtidas a partir do modelamento teórico do problema através das expressões em (43) até (45). Na determinação das assíntotas da função densidade espectral da diferença de fase, $W_{\Delta s}\left(k_{\uparrow}\right.$, $\left.k_{2}, \omega\right)$, foi empregada ainda a relação [5]

$$
W_{\Delta S}\left(k_{1}, k_{2}, \omega\right)=W_{S}\left(k_{1}, k_{1}, \omega\right)+W_{S}\left(k_{2}, k_{2}, \omega\right)-2 W_{S}\left(k_{1}, k_{2}, \omega\right)
$$

Observa-se uma boa concordância entre os espectros teórico e experimental para as log-amplitudes em 36,1 e $55,5 \mathrm{GHz}$. No caso dos espectros da diferença de fase, ernbora a limitada faixa de freqüências de flutuação medidas 
VARIÅNCIAS

\begin{tabular}{|c|c|c|c|c|c|c|c|c|c|}
\hline $\begin{array}{c}\text { Número } \\
\text { do } \\
\text { Experimento }\end{array}$ & Hora & $\begin{array}{l}\text { Banda Lateral } \\
\text { Superior } \\
\left(\mathrm{dB}^{2}\right)\end{array}$ & $\begin{array}{c}55 \mathrm{GHz} \\
\text { Portadora } \\
\text { (dB') }\end{array}$ & $\begin{array}{c}\text { Banda Lateral } \\
\text { Superior } \\
\left(\mathrm{dB}^{2}\right)\end{array}$ & $\begin{array}{c}36 \mathrm{GHz} \\
\text { Portadora } \\
\text { (dB2) }\end{array}$ & $\begin{array}{c}\text { Diferença } \\
\text { de Fase } \\
\text { ( rad2) }\end{array}$ & $\begin{array}{c}\text { Temperatura } \\
\left(K^{2}\right)\end{array}$ & $\begin{array}{l}\text { Pressăo de } \\
\text { Vapor d’ăgua } \\
\left.\text { (mb } \text { mb }^{2}\right)\end{array}$ & $\begin{array}{c}\text { Covariância } \\
\text { (K.mb) }\end{array}$ \\
\hline $\begin{array}{c}1 \\
2 \\
3 \\
4 \\
5 \\
6 \\
7 \\
8 \\
9 \\
10 \\
11 \\
12\end{array}$ & $\begin{array}{l}16: 00 \\
1430 \\
10: 00 \\
1830 \\
1930 \\
16: 45 \\
17300 \\
18200 \\
1230 \\
1400 \\
1230 \\
1300\end{array}$ & $\begin{array}{c}\bar{z} \\
= \\
= \\
= \\
= \\
\overline{-} \\
222 \mathrm{E}-2 \\
8,31 \mathrm{E}-3\end{array}$ & $\begin{array}{c}8,70 \mathrm{E}-2 \\
1,96 \mathrm{E}-2 \\
3,24 \mathrm{E}-2 \\
3,83 \mathrm{E}-2 \\
1,96 \mathrm{E}-2 \\
= \\
= \\
= \\
= \\
=\end{array}$ & $\begin{array}{c}\overline{ } \\
\bar{z} \\
= \\
\bar{z} \\
\bar{z} \\
2,31 \mathrm{E}-2 \\
8,47 \mathrm{E}-3\end{array}$ & $\begin{array}{l}1,35 \mathrm{E}-2 \\
1,02 \mathrm{E}-2 \\
1,77 \mathrm{E}-2 \\
1,96 \mathrm{E}-2 \\
5,90 \mathrm{E}=3 \\
1,08 \mathrm{E}-2 \\
1,26 \mathrm{E}-2 \\
6,36 \mathrm{E}-2 \\
5,18 \mathrm{E}=3 \\
7,06 \mathrm{E}=3 \\
1,12 \mathrm{E}-2 \\
2,83 \mathrm{E}-3\end{array}$ & $\begin{array}{c}\bar{z} \\
\bar{z} \\
\bar{z} \\
5,76 \mathrm{E}-5 \\
8,90 \mathrm{E}-5 \\
6,34 \mathrm{E}-4 \\
4,61 \mathrm{E}-5 \\
2,78 \mathrm{E}-5 \\
7,16 \mathrm{E}-6 \\
4,57 \mathrm{E}-5\end{array}$ & $\begin{array}{l}9,32 \mathrm{E}-2 \\
7,45 \mathrm{E}-2 \\
7,77 \mathrm{E}-2 \\
5,90 \mathrm{E}-2 \\
1,21 \mathrm{E}-2 \\
4,17 \mathrm{E}-2 \\
1,85 \mathrm{E}-2 \\
4,88 \mathrm{E}-2 \\
2,37 \mathrm{E}-1 \\
1,81 \mathrm{E}-2 \\
2,19 \mathrm{E}-1 \\
2,50 \mathrm{E}-2\end{array}$ & $\begin{array}{l}1,23 \mathrm{E}-3 \\
3,34 \mathrm{E}-3 \\
1,62 \mathrm{E}-2 \\
7,88 \mathrm{E}-3 \\
2,17 \mathrm{E}-3 \\
2,49 \mathrm{E}=2 \\
4,98 \mathrm{E}-3 \\
4,31 \mathrm{E}-2 \\
3,48 \mathrm{E}=2 \\
1,24 \mathrm{E}=2 \\
4,38 \mathrm{E}-2 \\
2,90 \mathrm{E}-3\end{array}$ & $\begin{array}{l}1,48 \mathrm{E}-2 \\
1,02 \mathrm{E}-2 \\
3,78 \mathrm{E}-2 \\
2,15 \mathrm{E}-2 \\
4,87 \mathrm{E}-3 \\
2,80 \mathrm{E}-2 \\
6,75 \mathrm{E}-3 \\
2,27 \mathrm{E}-2 \\
8,32 \mathrm{E}-2 \\
9,27 \mathrm{E}-3 \\
1,06 \mathrm{E}-1 \\
8,35 \mathrm{E}-3\end{array}$ \\
\hline
\end{tabular}

CONSTANTES DE ESTRUTURA

MÉDIAS

\begin{tabular}{|c|c|c|c|c|c|c|c|c|}
\hline $\begin{array}{c}\text { Número } \\
\text { do } \\
\text { Experimento }\end{array}$ & Hora & Condiçఠes do Tempo & $c_{n}^{2}$ & $c_{m}^{2} / c_{n}^{2}$ & $c_{n m} / c_{n}^{2}$ & $\underset{(\mathrm{m} / \mathrm{s})}{(\mathrm{V})}$ & $\begin{array}{l}(T) \\
(C)\end{array}$ & $\begin{array}{c}(\theta) \\
(\mathrm{mb})\end{array}$ \\
\hline $\begin{array}{r}1 \\
2 \\
3 \\
4 \\
5 \\
6 \\
7 \\
8 \\
9 \\
10 \\
11 \\
12\end{array}$ & $\begin{array}{l}16: 00 \\
14: 30 \\
10200 \\
1830 \\
1930 \\
16: 45 \\
17: 00 \\
18000 \\
1230 \\
14: 00 \\
1230 \\
13500\end{array}$ & $\begin{array}{c}\text { nublado } \\
\text { nublado } \\
\text { sol } \theta \text { frio } \\
\text { neblina } \\
\text { nublado e quente } \\
\text { sol } \\
\text { sol } \\
\text { nublado e quente } \\
\text { nublado e quente } \\
\text { nublado } \\
\text { sol } \theta \text { frio } \\
\text { sol }\end{array}$ & $\begin{array}{l}6,09 \mathrm{E}-14 \\
4,60 \mathrm{E}-14 \\
7,98 \mathrm{E}-14 \\
8,84 \mathrm{E}-14 \\
2,66 \mathrm{E}-14 \\
4,87 \mathrm{E}-14 \\
5,68 \mathrm{E}-14 \\
2,87 \mathrm{E}-14 \\
2,34 \mathrm{E}-14 \\
3,18 \mathrm{E}-14 \\
5,07 \mathrm{E}-14 \\
1,26 \mathrm{E}-14\end{array}$ & $\begin{array}{l}8,54 \mathrm{E}-5 \\
8,03 \mathrm{E}-6 \\
3,78 \mathrm{E}-5 \\
6,33 \mathrm{E}-5 \\
1,02 \mathrm{E}-5 \\
2,68 \mathrm{E}-6 \\
4,96 \mathrm{E}-6 \\
1,1 \mathrm{ME}-6 \\
2,22 \mathrm{E}-6 \\
9,10 \mathrm{E}-6 \\
9,28 \mathrm{E}-5 \\
6,37 \mathrm{E}-5\end{array}$ & $\begin{array}{l}5,77 \mathrm{E}-2 \\
5,09 \mathrm{E}-3 \\
3,60 \mathrm{E}-2 \\
5,56 \mathrm{E}-2 \\
1,06 \mathrm{E}-2 \\
2,12 \mathrm{E}-3 \\
3,16 \mathrm{E}-3 \\
6,24 \mathrm{E}-4 \\
1,39 \mathrm{E}-2 \\
7,88 \mathrm{E}-4 \\
8,78 \mathrm{E}-2 \\
525 \mathrm{E}-2\end{array}$ & $\begin{array}{l}8,0 \\
3,0 \\
2,0 \\
1,5 \\
3,5 \\
3,0 \\
2,5 \\
3,5 \\
3,0 \\
1,0 \\
1,0 \\
2,5\end{array}$ & $\begin{array}{r}5,0 \\
4,5 \\
5,2 \\
5,6 \\
21,0 \\
13,7 \\
13,2 \\
18,9 \\
17,3 \\
7,7 \\
3,8 \\
10,2\end{array}$ & $\begin{array}{l}86,1 \\
85,1 \\
79,5 \\
59,0 \\
65,0 \\
= \\
= \\
\overline{-} \\
81,0 \\
92,0 \\
61,0\end{array}$ \\
\hline
\end{tabular}

Tabela 1. Variâncias e médlas observadas $\theta$ constantes de estrutura calculadas para os

diversos experimentos. 

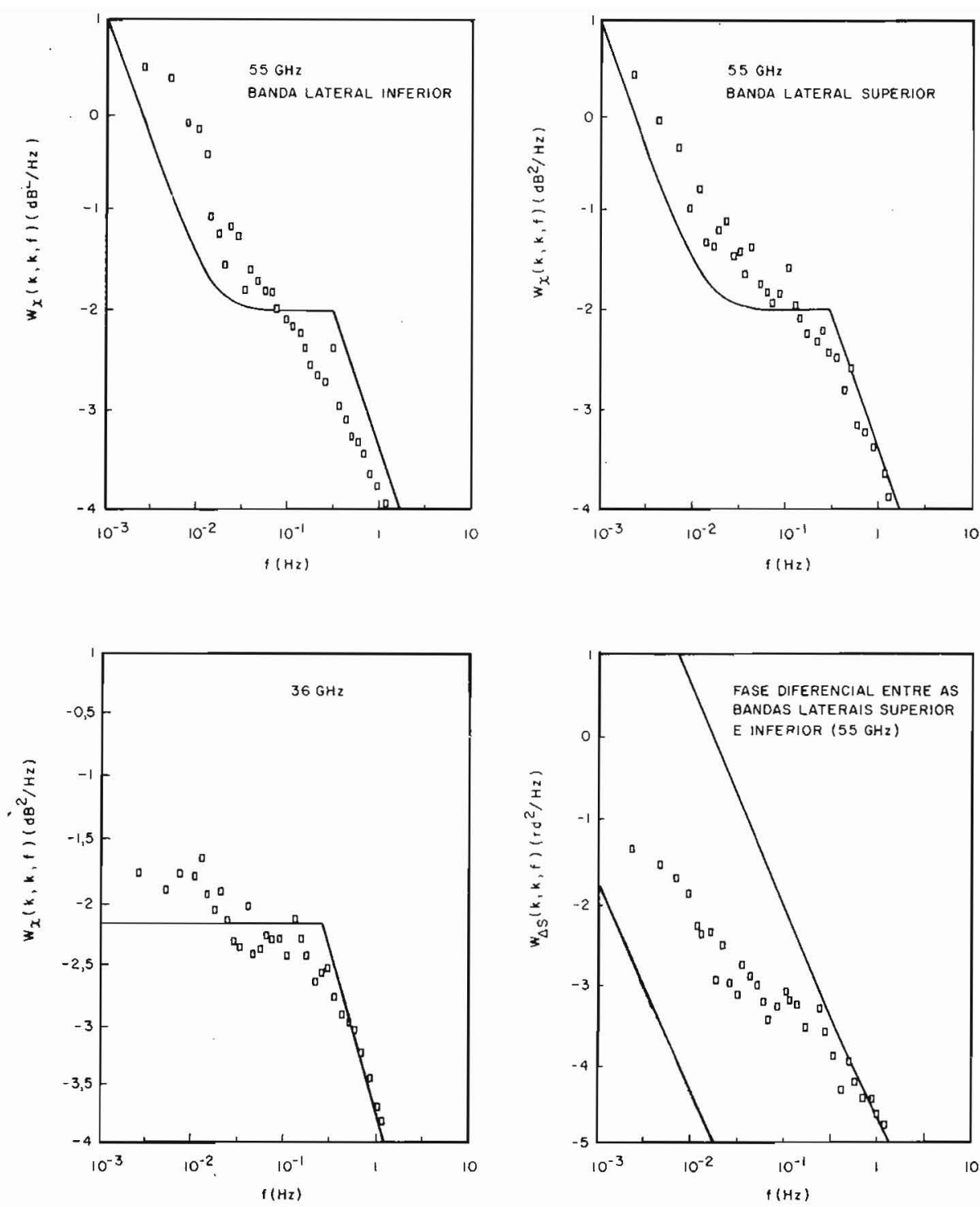

Figura 5. Funçð̃es densidade espectral temporal obtıdas no experimento 12.

não permita uma verificação completa da tendência assintótica dos dados experimentais na região de baixas freqüências, estes dados apresentam um comportamento coerente com os resultados téricos na região de altas freqũências. 


\section{Conclusões}

Foi desenvolvido um modelo teórico para determinação das funçōes densidade espectral das flutuações temporais da log-amplitude e da fase do sinal recebido em enlaces em ondas milimétricas operando em faixas de freqüências sujeitas à absorção por gases em condições de turbulência. O modelo reproduz com exatidão resultados teóricos anteriores, obtidos para freqüências em que o mecanismo de absorção pode ser desprezado, constituindo-se numa solução geral que engloba esta situação como caso particular.

É possivel concluir que o mecanismo de absorçã̃o provoca um aumento na intensidade das flutuações lentas da amplițde do sinal recebido $\mathrm{em}$ condições de turbulência atmosférica. Este fato se reflete no crescimento da função densidade espectral de log-amplitude quando a freqüência de flutuação tende para zero, no caso em que o mecanismo de absorção é efetivo, em contraposição ao patamar nesta função, na região de baixas freqüências de flutuação, quando o efeito de absorção é desprezivel.

Para validar este modelo teórico, foram iealizadas medidas do sinal recehido em dois enlaces experimentais, operando nas faixas de freqüencias de 36 e $55 \mathrm{GHz}$, e de parâmetros meteorológicos num dos extremos destes enlaces. Os dados experimentais mostraram, em geral, boa concordância corn o modelo ieórico.

\section{Referências}

[1] A. D. Sayers, "Radiometric Sky Temperature Measurements at 35 and

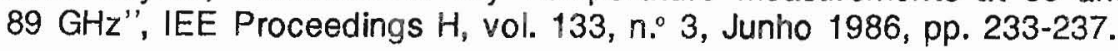

[2] G. G. Haroules e W. E. Brown, "A $60 \mathrm{GHz}$ Multi-Frequency Radiometric Sensor for Detecting Clear Air Turbulence in the Troposphere", IE Transactions on Aerospace and Electronic Systems, vol. AES-5, n..5, 1969, pp. 712-723.

[3] J. W. Waters, K.F. Kunzi, R.L. Pettyjohn, R.K.L. Poon e D.H. Staelin, "Fiemote Sensing of Atmospheric Temperatuie Profiles with the Nimbus 5 Microwave spectrometer", Journal of Atmospheric Science, vol. 32 , 1975, pp. 1953-1969.

[4] R.K.L. Poon, "A Power Law Fit to Oxygen Absorption at $60 \mathrm{GHz}$ and is Application to Remote Sensing of Atmospheric Temperature", Radio Science, vol. 15, n. 1, Janeiro-Fevereiro 1980, pp. 25-33.

[0] R. Woo. A. Ishimaru e W.B. Kendall, "Observations of Small-Scale Turbulence in the.Atmosphere of Venus by Mariner 5", Journal of Atmospheric Science, vol. 31, n. 6,1974, pp. 1698-1706. 
[6] L.A. Chernov, "Wave Propagation in a Random Medium", McGraw Hill, New York, 1960.

[7] V.I. Tatarski, "Wave Propagation in a Turbulent Medium", Dover Publications, Inc., New York, 1967.

[8] V.I Tatarski, "The Effects of the Turbulent Atmosphere on Wave Propagation", NTIS, Springfield, 1971.

[9] A Ishimaru, "Temporal Frequency Spectra of Multifrequency Waves in Turbuient Atmosphere", IEEE Transactions on Antennas and Propagation, vol. AP-20, n.91, Janeiro 1972, pp. 10-19.

[10] A. Ishimaru, "Wave Propagation and Scattering in Random Media", Academic Press, New York, 1978.

[11] A. Ishimaru, "Fluctuations of a Beam Wave Propagating Through a Locally Homogeneous Medium", Radio Science, vol. 4, n. 4, Abril 1969, pp. 295-305.

[12] A.O. Izyumov, "Amplitude and Phase Fluctuations of a Plane Monochromatic Wave in a Neai-Ground Layer of Moisture Containing Turbulent Air", Radio Eng. Electron. Phys., vol 13, n. 7, 1968, pp. 1009-1013.

[13] A.S. Gurvich, "Effect of Absorption on the Fluctuation in Signal Level During Atmospheric Propagation", Radio Eng. Electron. Phys., vol. 13, n. ${ }^{\circ} 11,1968$, pp. 1687-1694.

[14] N.A. Amnand, A. O. Izyumov e A.V. Sokolov, "Fluctuatioir of Submillimeter Waves in a Turbu!ent Atmosphere", Radio Eng. Electron. Phys., vol. 16, 1971, pp. 1259-1266.

[15] R.H. Ott,"'Temporal Radio Frequency Spectra of Multi-Frequency Waves in a Turbulent Atmosphere Characteized by a Complex Refractive Incex", IEEE Transartions on Antennas and Propagation, vol. AP-25, n. 2, Março 1977, pp. 254-260.

[16] R.H. Ott e M.C Thompson, Jr., "Atmospheric Amplitude Spectra in an Absorption Region", IEEE Transactions on Antennas and Propagation, vol. AP-26, n. 2, Março 1978, pp. 329-332.

[17] R.M. Manning, L.F. Merat e P.C. Claspy, "Theoretical Investigation of Millimeter Wave Propagation Through Clear Atmosphere", Millimeter Wave Tecinnology, Society of Photo-Optical Instrumentation Engineers, vol. 337,1982 , pp. 67-80. 
[18] H.B. Janes, M.C. Thompson, Jr., D. Smith e A.W. Kirkpatrick, "Comparison of Simultaneous Line-of-sight Signals at 9.6 and $34.52 \mathrm{GHz}$ ", IEEE Transactions on Antennas and Propagation, vol. AP-18, n. 4, Julho 1970, pp. $447-451$.

19] K.L. Ho, N.D. Mavrokoukoulakis e R.S Cole, "Propagation Studies on a Line-of-Sight Microwave Link at $36 \mathrm{GHz}$ and $110 \mathrm{GHz}$ ", Microwaves, Optics and Acoustics, vol. 3, n. 3 , 1979, pp. 93-98.

20] N.A. Armand, A.O. Izyumov, B.I. Polevoy, A.V. Sokolov e A.I. Topkov, "Fluctuation of Millimeter Radio Waves Propagated Through a Turbulent Atmosphere Near the Oxygen Absorption Line Centered at the Wavelength of 5mm", Radio Eng. Electron. Phys., vol. 18, n. 4, 1973, pp. 492-496.

21] D.A.R. Jayasuriya, F.C. Medeiros Filho e R.S. Cole, "Scintillation Fading in an Absorption Region", IEE Second International Conference on Antenas and Propagation, Inglaterra, 1981, pp. 221-224.

22] F.C. Medeiros Filho, D.A.R. Jayasuriya e R.S. Cole, "Spectral Density of Amplitude Scintillation on a $55 \mathrm{GHz}$ Line-of-Sight Link", Electronics Letters, vol. 17, n. ${ }^{\circ}$, Janeiro 1981, pp. 25-26.

23] R.H. Ott e M.C Thompson, Jr., "Characteristics of a Radio Link in the 55 to $65 \mathrm{GHz}$ Range", IEEE Transactions on Antennas and Propagation, vol. AP-26, Novembro 1976, pp. 873-877.

24] A.N. Kolmogorov, "The Local Structure of Turbulence in Incompressible Viscous Fluid for Very Large Reynolds Numbers", Doklady Akad. Nauk SSSK, 30, 301, 1941.

25] R.J. Hill, S.F. Clifford e R.S Lawrence, "Refractive-Index and Absorption Fluctuations in the Infrared Caused by Temperature, Humidity, and Pressure Fluctuations", Journal of the Optical Society of America, vol. 70, n. 10,1980 , pp. 1192-1205.

26] F.C. Medeiros Filho, "Millimetre Wave Propagation in an Absorption Region", Ph.D. Thesis, University College London, 1981.

27] L.A.R. Silva Mello, "Efeitos de Cintilação em Ondas Milimétricas ná Faixa de Absorção do Oxigênio", Tese de Doutorado, Departamento de Engenharia Elétrica, PUC/Rio, Maio 1987.

38] M. Abramowitz e I.A. Stegun, "Handbook of Mathematical Functions", Dover Publications, Inc., New York. 


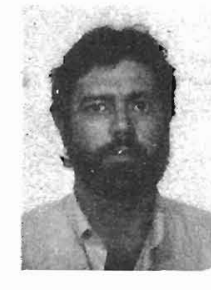

LUIZ A. R. DA SILVA MELLO nasceu no Rio de Janeiro em 1. de maio de 1953. Formou-se em Engenharia Elétrica-Telecomunicações na PUC/Rio, em 1975, e obteve os graus de Mestre om Ciências e Doutor em Ciências em Engenharia Elétriça, na mesma Universidade, em 1977 e 1987, respectivamente. E Professor da PUC/Rio desde 1978, exercendo atividades de pesquisa no Centro de Estudos em Telecomunicações da Universidade Católica (CETUC). Suas áreas de interesse são o eletromagnetismo aplicado e a propagação de ondas radioelétricas.

CARLOS GUSTAVO S. MIGLIORA, fotografia e biografia podem ser encontradas neste número; p. 23. 